Results: Ocular involvement was found in 114 (27\%) of 417 GPA patients registered in POLVAS, 34 (8\%) developed orbital masses. Mean patients' age was 47.8 (range from 19-75) yrs., $23(67 \%)$ were women. Forty four per cent of the patients developed tumor at the beginning of the disease, $56 \%$ during relapse. Patients' characteristics on diagnosis of orbital mass: 24 cANCA, 2 pANCA, and 8 ANCA negative, $9 \%$ active smokers and $31 \%$ past smokers, $29 \%$ had localized disease, $21 \%$ early systemic and $50 \%$ systemic with organ involvement, $29 \%$ had other type of ophthalmological involvement before pseudotumor occurred, $88 \%$ had active paranasal sinus involvement, $41 \%$ lungs, $15 \%$ CNS, $15 \%$ skin and $6 \%$ heart manifestations. Thirty seven per cent of patients had positive nasal swabs cultures, $50 \%$ of which were positive for Staphylococcus aureus. In $65 \%$, tumor occurred during steroid therapy ( $46 \%$ had prednisone more than $5 \mathrm{mg} / \mathrm{d}$ ) and $45 \%$ on immunosuppressive treatment (19\% when treated with AZA, $16 \%$ MTX, 6,5\% MMF and 3,5\% CYC). Due to orbital mass $86.5 \%$ were treated with CYC and $13.5 \%$ with RTX. Twenty one per cent had complete remission of the pseudotumor, $76 \%$ partial remission and in $3 \%$ patients there was no response to the treatment; $43 \%$ developed visual impairment, $20 \%$ suffered from blindness. Conclusion: Orbital inflammatory mass was not common manifestation of GPA among our patients. The mass developed at the beginning or in the course of the disease, even during immunosuppressive treatment. Orbital masses have been resistant to therapeutic interventions and were accompanied by high risk of visual impairment.

Disclosure of Interests: Anna Masiak: None declared, Marcin Ziętkiewicz: None declared, Krzysztof Wójcik: None declared, Katarzyna Wawrzycka-Adamczyk: None declared, Radoslaw Jeleniewicz: None declared, Marta Madej: None declared, Joanna Kur-Zalewska: None declared, Katarzyna Jakuszko: None declared, Małgorzata Wisłowska: None declared, Hanna Storoniak: None declared, Michał Komorniczak: None declared, Barbara Bułło-Piontecka: None declared, Iwona Brzosko: None declared, Małgorzata Stasiek: None declared, Eugeniusz Kucharz: None declared, Alicja Dębska-Ślizień: None declared, Maria Majdan Consultant of: Roche, Amgen, Speakers bureau: Roche, Amgen, Jacek Musiał: None declared, Zbigniew Zdrojewski: None declared DOI: 10.1136/annrheumdis-2020-eular.2881

\section{FRI0211 VASCULITIS ASSOCIATED WITH MYELODYSPLASTIC SYNDROME AND CHRONIC MYELOMONOCYTIC LEUKEMIA: FRENCH MULTICENTER CASE CONTROL STUDY}

A. Roupie ${ }^{1}$, B. Terrier ${ }^{2}$, P. Fenaux ${ }^{1}$, O. Fain ${ }^{1}$, A. Mekinian ${ }^{1}$ on behalf of Minhemon. ${ }^{1}$ Sorbonne University, Internal Medicine Department, Saint Antoine Hospital, Paris, France; ${ }^{2}$ Paris Descartes V University, Internal Medicine Department, Saint Antoine Hospital, Paris, France

Background: Myelodysplastic syndromes (MDS) and MDS/myeloproliferative neoplasms (MDS/MPN) can be associated with vasculitis.

Objectives: In this nationwide study by the "French Network of dysimmune disorders associated with hemopathies" (MINHEMON) the objective was to evaluate characteristics, treatment and outcome of vasculitis MDS-MDS/MPN

Methods: Retrospective analysis of patients that presented a MDS/MPN associated with vasculitis and compared the overall survival and acute leukemia with MDS without vasculitis.

Results: Seventy patients with vasculitis and MDS/MPN were included, with a median age of 71.5 [21-90] years and male/female ratio of 2.3. Vasculitis was diagnosed prior to MDS/MPN in 31 patients (44.3\%), with a median time of 27 months [1-120] between two diagnosis, and after in 20 patients ( 6 months [1-59]). In comparison to $183 \mathrm{MDS} / \mathrm{MPN}$ without dysimmune features showed no difference in MDS/MPN subtypes distribution nor median IPSS/CPSS scores in patients with and without vasculitis. The vasculitis subtypes was giant-cell arteritis (GCA) in 24 patients $(34 \%)$. Eleven patients $(20 \%)$ had Behçet's-like syndrome and 6 patients $(9 \%)$ presented with polyarteritis nodosa. Steroids $(60 \mathrm{mg} /$ day $[0-500]$ of prednisone equivalent) were used as first-line therapy for MDS/MPN vasculitis in 64/70 patients $(91 \%)$ and $41(59 \%)$ received combined immunosuppressive therapies during the follow-up. After the follow-up of 33.2 months [1-162], 31 patients (44\%) finally experienced sustained remission. At least one relapse during the 33.2 months [1-162] follow-up occurred in 43 patients $(61 \%)$. Relapse rates were higher in patients treated by DMARDs (odds ratio at 4.86 [ $95 \% \mathrm{Cl} 1.38-17.10]$ ), but did not differ from biologics (odds ratio 0.59 [95\% $\mathrm{Cl} 0.11-3.20]$ ) and azacytidine (odds ratio 1.44 [95\% $\mathrm{Cl} 0.21-9.76]$ ) (steroids considered as reference). Overall survival and progression to acute myeloid leukemia in MDS/MPN vasculitis were not significantly different from MDS/MPN patients without any dysimmune features $(p=0.5)$.

Conclusion: This first largest study of MDS/MPN vasculitis show no correlation of vasculitis subtypes with various subtypes and severity of MDS/MPN, and no significant impact of vasculitis on overall survival and progression to acute myeloid leukemia. The high relapse rats and steroid dependence raise the question of combined therapies to steroids. Whereas DMARDs use seem to be avoid specific azacytidine therapy could be considered for even low-risk MDS/MPN vasculitis.
Acknowledgments: minhemon gfm gfev Disclosure of Interests: None declared DOI: 10.1136/annrheumdis-2020-eular.4485

\section{FRI0212 \\ THE ROLE OF AGE ON THE CLINICAL PRESENTATION AND RELAPSE RATES IN A LARGE COHORT OF 720 PATIENTS WITH GIANT CELL ARTERITIS}

S. Monti ${ }^{1}$, L. Dagna ${ }^{2}$, C. Campochiaro ${ }^{2}$, A. Tomelleri ${ }^{2}$, G. Zanframundo ${ }^{1}$, C. Klersy ${ }^{3}$, F. Muratore ${ }^{4}$, L. Boiardi' ${ }^{4}$, R. Padoan ${ }^{5}$, M. Felicetti ${ }^{5}$, F. Schiavon 6 , M. Bond ${ }^{7}$, A. Berti ${ }^{7}$, R. Bortolotti ${ }^{7}$, C. Nannini ${ }^{8}$, F. Cantini $i^{8}$, A. Giollo ${ }^{9}$, E. Conticini ${ }^{10}$, A. Gattamelata ${ }^{11}$, R. Priori' ${ }^{11}$, L. Quartuccio ${ }^{12}$, E. Treppo ${ }^{12}$, G. Emmi ${ }^{13}$, M. Finocchi ${ }^{13}$, G. Cassone ${ }^{14}$, A. Hoxha ${ }^{15}$, R. Foti ${ }^{16}$, M. Colaci ${ }^{16}$, R. Caporali ${ }^{17}$, C. Salvarani ${ }^{18}$, C. Montecucco ${ }^{1}$ on behalf of Italian Society of Rheumatology Vasculitis Study Group. ${ }^{1}$ Pavia, Rheumatology, IRCCS Fondazione Policlinico S. Matteo, Pavia, Italy; ${ }^{2}$ Milan, Immunology, VitaSalute San Raffaele University, Milan, Italy; ${ }^{3}$ Pavia, Biometry and Clinical Epidemiology, IRCCS Fondazione Policlinico S. Matteo, Pavia, Italy; ${ }^{4}$ Reggio Emilia, Rheumatology Unit, AUSL-IRCCS di Reggio Emilia, Reggio Emilia, Italy; ${ }^{5}$ Padova, Rheumatology, Padua, Italy; ${ }^{6}$ Padua, Rheumatology, Padua, Italy; ${ }^{7}$ Trento, Rheumatology, Trento, Italy; ${ }^{8}$ Prato, Rheumatology, Prato, Italy ${ }^{9}$ Verona, Rheumatology, Verona, Italy; ${ }^{10}$ Siena, Rheumatology, Siena, Italy;

${ }^{11}$ Rome, Policlinico Umberto I, Rome, Italy; ${ }^{12}$ Udine, Rheumatology, Udine, Italy; ${ }^{13}$ Florence, Immunology, Florence, Italy; ${ }^{14}$ Modena, Rheumatology, Modena, Italy; ${ }^{15}$ Vicenza, Ospedale San Bortolo, Vicenza, Italy; ${ }^{16}$ Catania, Rheumatology, Catania, Italy; ${ }^{17}$ Milan, Rheumatology, Ist. Pini, Milan, Italy; ${ }^{18}$ Reggio Emilia, Rheumatology Unit, AUSL-IRCCS di Reggio Emilia, University of Modena and Reggio Emilia, Reggio Emilia, Italy

Background: Giant cell arteritis (GCA) is the most frequent systemic vasculitis after the age of 50 years old. Recent interest in the processes of immune and vascular aging have been proposed as a disease risk factor. Data on the impact of age at diagnosis of GCA on the clinical course of the disease are scarce Objectives: To assess the role of age at diagnosis of GCA on the risk and time to relapse

Methods: Centres participating in the Italian Society of Rheumatology Vasculitis Study Group retrospectively enrolled patients with a diagnosis of GCA until December 2019. The cohort was divided in tertiles according to age at diagnosis ( $\leq 72 ; 73-79 ;>79$ years old). Negative binomial regression was used to assess the relapse rate according to age groups, and Cox regression for time to first relapse.

Results: Of 720 patients enrolled in 14 Italian reference centres, 711 had complete follow-up data (female 50\%; mean age $75 \pm 7$ ). Median follow-up duration was 34 months (IQR 16;70). Patients in the older group at diagnosis (> 79 years) had more frequent visual loss compared to the $73-79$ and $\leq 72$ age groups $(31 \%$ vs $20 \%$ vs $7 \%$; $p<0.001)$, but lower rates of general symptoms $(56 \%$ vs $70 \%$ vs $77 \% ; p<0.001)$. Large-vessel (LV)-GCA was less frequent in the older group ( $18 \%$ vs $22 \%$ vs $43 \%$; $p<0.001$ ). At least one relapse occurred in $47 \%$ of patients. Median time to relapse was 12 months (IQR 6;23). Age did not influence the rate of relapses [18 per 100 persons/years $(95 \% \mathrm{Cl} 15 ; 21)$ vs $19(95 \% \mathrm{Cl} 17 ; 22)$ vs 19 $(95 \% \mathrm{Cl} 17 ; 22)]$, nor the time to first relapse (Figure 1). LV-GCA, presentation with significantly elevated c-reactive protein (>50 mg/L) and general symptoms were independent predictors of relapse.

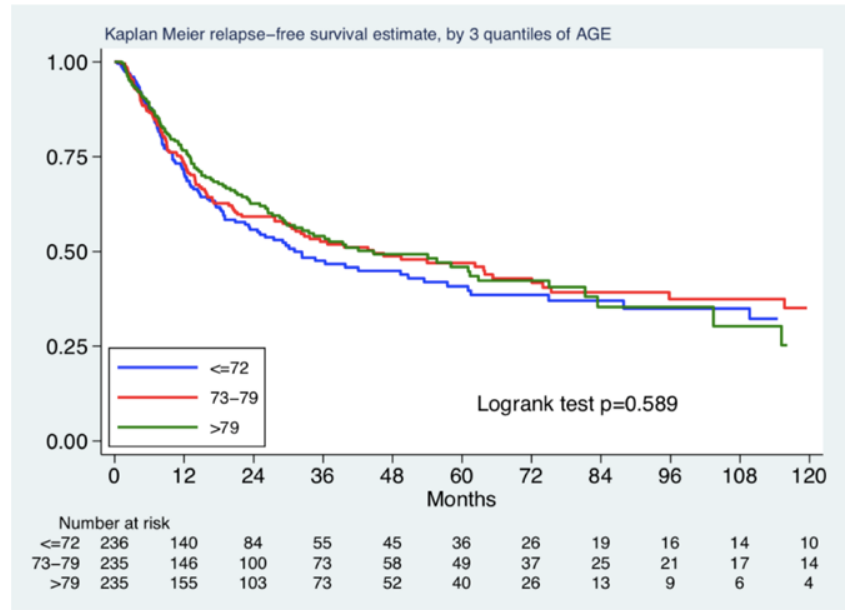

Conclusion: Age at diagnosis of GCA influenced the clinical presentation and risk of ischaemic complications, but did not affect the relapse rate during follow-up. LV-GCA occurred more frequently in younger patients and was an 
independent predictor of relapse risk, highlighting the need for a correct characterization of the clinical subtype at the early stages of disease.

Disclosure of Interests: Sara Monti: None declared, Lorenzo Dagna Grant research support from: Abbvie, BMS, Celgene, Janssen, MSD, Mundipharma Pharmaceuticals, Novartis, Pfizer, Roche, SG, SOBI, Consultant of: Abbvie, Amgen, Biogen, BMS, Celltrion, Novartis, Pfizer, Roche, SG, and SOBI, Corrado Campochiaro Speakers bureau: Novartis, Pfizer, Roche, GSK, SOBI, Alessandro Tomelleri: None declared, Giovanni Zanframundo: None declared, Catherine Klersy: None declared, Francesco Muratore: None declared, Luigi Boiardi: None declared, Roberto Padoan: None declared, Mara Felicetti: None declared, Franco Schiavon: None declared, Milena Bond: None declared, Alvise Berti: None declared, Roberto Bortolotti: None declared, Carlotta Nannini: None declared, Fabrizio Cantini: None declared, Alessandro Giollo: None declared, Edoardo Conticini: None declared, angelica gattamelata: None declared, Roberta Priori: None declared, Luca Quartuccio Consultant of: Abbvie, Bristol, Speakers bureau: Abbvie, Pfizer, Elena Treppo: None declared, Giacomo Emmi: None declared, Martina Finocchi: None declared, Giulia Cassone: None declared, Ariela Hoxha Speakers bureau: Celgene, UCB, Novartis, Sanofi, Werfen, Rosario Foti Consultant of: lilly, sanofi, MSD, Janssen, Abbvie, BMS, celgene, roche, Speakers bureau: lilly, sanofi, MSD, Janssen, Abbvie, BMS, celgene, roche, Michele Colaci: None declared, Roberto Caporali Consultant of: AbbVie; Gilead Sciences, Inc.; Lilly; Merck Sharp \& Dohme; Celgene; Bristol-Myers Squibb; Pfizer; UCB, Speakers bureau: Abbvie Bristol-Myers Squibb; Celgene; Lilly; Gilead Sciences, Inc; MSD; Pfizer; Roche; UCB, Carlo Salvarani: None declared, Carlomaurizio Montecucco: None declared DOI: 10.1136/annrheumdis-2020-eular.3779

\section{FRI0213 \\ ASSOCIATION BETWEEN SPECIMEN LENGTH AND NUMBER OF SECTIONS AND DIAGNOSTIC YIELD OF TEMPORAL ARTERY BIOPSY: A RETROSPECTIVE, SINGLE CENTER EXPERIENCE OVER A 21 YEARS' PERIOD}

F. Muratore ${ }^{1}$, L. Boiardi ${ }^{1}$, A. Cavazza ${ }^{2}$, T. Giacomo ${ }^{3}$, R. Aldigeri ${ }^{4}$, L. Cimino ${ }^{5}$, C. Salvarani $6 .{ }^{1}$ Division of Rheumatology, Azienda Unita' Sanitaria Locale IRCCS di Reggio Emilia, Reggio Emilia, Italy; ${ }^{2}$ Pathology Unit, Azienda Unita' Sanitaria Locale IRCCS di Reggio Emilia, Reggio Emilia, Italy; ${ }^{3}$ Division of Rheumatology, University of Modena and Reggio Emilia, Modena, Italy; ${ }^{4}$ Department of Medicine and Surgery, University of Parma, Parma, Italy, Parma, Italy; ${ }^{5}$ Ophthalmology Unit, Azienda Unita' Sanitaria Locale IRCCS di Reggio Emilia, Reggio Emilia, Italy; ${ }^{6}$ Division of Rheumatology, Azienda Unita' Sanitaria Locale IRCCS di Reggio Emilia and University of Modena and Reggio Emilia, Reggio Emilia, Italy

Background: Temporal artery biopsy (TAB) showing inflammation is considered the gold standard for the diagnosis of giant cell arteritis (GCA). However, sampling error may lead to a negative $T A B$, and a negative $T A B$ does not rule out GCA. The diagnostic sensitivity of TAB can be affected by the discontinuous character of the histopathologic changes (skip lesions) and by the length of specimens. The optimal TAB length and the optimal number of sections that need to be evaluated in order to avoid missing skip lesions are controversial.

Objectives: To investigate the association between specimen length and number of section and the diagnostic yield of TAB for GCA.

Methods: A pathologist with expertise in vasculitis and blinded to clinical data and final diagnosis reviewed all TABs performed for suspected GCA at our hospital between January 1991 and December 2012. The biopsies were routinely fixed in formalin and completely embedded in paraffin. Sections of 4 microns thickness were cut from paraffin blocks and stained with hematoxylin-eosin. TABs were classified into three categories: inadequate, when the biopsy did not sample the muscular artery; negative when the temporal artery was devoid of inflammation and positive when the temporal artery showed inflammation, arbitrarily defined as at least 1 aggregate of at least 15 inflammatory cells. The blocks of all the inadequate and negative biopsies were recut, and at least three further slides at deeper levels were stained with hematoxylin-eosin.

Results: 694 TABs were performed in the study period and were reviewed. 32 $(4.6 \%)$ were classified as inadequate and were excluded from the analysis. Of the remaining 662 TABs [71\% female; mean (SD) age, 73.2 (8.8) years], mean (SD) post fixation length was $6.63(4.42) \mathrm{mm}$, and median number of sections evaluated was 3 (range 1-33). 382 (58\%) TABs were classified as negative and $280(42 \%)$ as positive. Compared with negative TAB, patients with positive TAB were older [mean age (SD) $74(7.5)$ years vs $72(9.6), p=0.009$ ] and there was a trend for female predominance $(75 \%$ vs $68 \%, p=0.077)$. Post fixation length of the specimens was significantly lower in negative compared with positive TAB [mean (SD) $6.37(4.26) \mathrm{mm}$ vs 6.99 (4.61) respectively, $\mathrm{p}=0.026$ ]. Piecewise logistic regression identified $5 \mathrm{~mm}$ as the TAB length change point for diagnostic sensitivity. Compared with TAB length of $<5 \mathrm{~mm}$, age- and sex-adjusted odds ratio for positive $\mathrm{TAB}$ in samples $\geq 5 \mathrm{~mm}$ long were 1.536 (95\% confidence interval, 1.108 to 2.130 )
The median (IQR) number of sections evaluated were 2 (1-3) for positive TAB and 4 (2-5) for negative TAB, p<0.0001. In 26/280 (9.3\%) positive TABs, the first section was negative, and the inflammation was detected only in deeper sections (the positive section was the second in 14 TABs, the third in 9 and the fourth in 3). In all 26 cases, inflammation detected in deeper section was not transmural, but limited to adventitial or periadventitial small vessels.

Conclusion: Our data confirm that a post fixation TAB length of at least $5 \mathrm{~mm}$ should be sufficient to make a histological diagnosis of inflamed temporal artery. According to our data, in order to avoid missing skip inflammatory lesions, at least 3 further sections at deeper levels should be cut and evaluated in all negative TABs.

Disclosure of Interests: None declared

DOI: 10.1136/annrheumdis-2020-eular.5485

\begin{tabular}{|l|l|}
\hline FRI0214 & PERSISTENT LOW-GRADE FDG-PET VASCULAR \\
INFLAMMATION IN REMITTED LVV-GCA PATIENTS \\
IS ASSOCIATED TO A SIGNIFICANT HIGH RISK OF \\
RELAPSE
\end{tabular}

R. Padoan ${ }^{1}$, A. Tomelleri ${ }^{2}$, M. Felicetti ${ }^{1}$, C. Campochiaro ${ }^{2}$, E. Baldissera ${ }^{2}$, F. Crimi ${ }^{3}$, P. Zucchetta ${ }^{4}$, D. Cecchin ${ }^{4}$, M. Picchio ${ }^{5}$, L. Dagna ${ }^{2}$, A. Doria ${ }^{1}$, F. Schiavon ${ }^{1} .{ }^{1}$ Rheumatology Unit, University of Padova, Padova, Italy; ${ }^{2}$ Unit of Immunology, Rheumatology, Allergy and Rare Diseases, IRCCS San Raffaele Hospital, Milano, Italy; ${ }^{3}$ Radiology Unit, University of Padova, Padova, Italy; ${ }^{4}$ Unit of Nuclear Medicine, University of Padova, Padova, Italy; ${ }^{5}$ Unit of Nuclear Medicine, IRCCS San Raffaele Hospital, Milano, Italy

Background: Persistent low-grade vascular inflammation in giant cell arteritis (GCA) with large vessel involvement (LVV) treated patients could represent the expression of persistent subclinical disease activity or post-inflammatory vascular remodelling. Whether these findings have any impact on future vascular outcomes is still an unmet need.

Objectives: To evaluate the frequency and evolution of FDG-PET low-grade vascular inflammation in remitted LVV-GCA patients.

Methods: We included all consecutive patients classified as GCA with LVV involvement, with a minimum disease duration of 12 months and clinically remitted, who underwent to at least one PET/MR scan between January 2015 and January 2020 For each scan vessel's metabolic activity was assessed using the Meller's grading ${ }^{1}$. Low-grade inflammation was defined as Meller 1 and 2 (inferior or equal to liver) as reported in previous studies. Demographic and clinical data, as well as disease remission or flares, were recorded and compared to vascular metabolic activity.

Results: In total 88 PET scans were performed in 54 LVV-GCA patients, predominantly female $(77.8 \%)$, aged $68[7,8]$ years, with a regular BMI (23.9[2.8]) and with a long-standing disease (27[32.6] months). A subsequent PET/MR scan was available in 34 patients (median time between the two scans 9[6.3] months).

At first PET examination, low-grade metabolic activity was reported in $68,5 \%$ of the cases, while complete remission in $15 \%$ and high metabolic activity in $25 \%$. Comparing patients with low-grade vascular inflammation to those with complete remission (Meller 0), they had lower disease duration (28[25.9] vs 73[68] months, but without significance) and they were treated with higher daily prednisone dosage $(5[3.8]$ vs $0[2.2], p=0.042)$. No significant differences were noted in age, acute phase reactants and type of treatment. Moreover, when compared to those with high metabolic activity (Meller 3 ), the latter had only significantly higher CRP levels (8.3[13.8] vs 4.1[3.9], $p=0.03$ ) and lower disease duration (19[20.6] vs 28[25.9] months, but without significance). While no significant differences were noted in age and type of treatment (both glucocorticoids and immunosuppressants)

Among all patients with low-grade vascular inflammation, $81 \%$ of them underwent to steroids or immunosuppressants tapering due to clinical remission. At the subsequent PET examination, a worsening of metabolic activity (Meller 3 ) was found in $4 / 20$ patients, with 1 clinical flare. While in 14/20 patients the subsequent PET revealed a persistent metabolic activity. Only in $2 / 20$ there was a complete metabolic remission. Change or increase of the treatment regimen led to an improvement (Meller 0 or 1 ) in all the cases. Low-grade metabolic activity was associated with a significant increased risk of worsening/flare at the subsequent PET examination (RR 5.29[1.87-16.11], $p=0.002$ )

Conclusion: Low-grade vascular inflammation at PET examination is a common feature in remitted patients. It is associated with older age, lower disease duration and clinical remission. Treatment tapering is associated with an increased risk of worsening/flare. Further research is urgently needed to address this issue.

References:

[1] J. Meller et al., "Early diagnosis and follow-up of aortitis with [(18)F]FDG PET and MRI.," Eur. J. Nucl. Med. Mol. Imaging, vol. 30, no. 5, pp. 730-6, May 2003.

Disclosure of Interests: Roberto Padoan: None declared, Alessandro Tomelleri: None declared, Mara Felicetti: None declared, Corrado Campochiaro 